\title{
Frecuencia de Procesos Patológicos en Canis Familiaris mediante el Diagnóstico de Imágenes de Rayos X en la Ciudad de Tacna
}

\section{Frequency of Pathological Processes in Canis Familiaris by Diagnosing $\mathrm{X}$-Ray Pictures in the City of Tacna}

\author{
${ }_{1}^{1}$ Hugo Flores Aybar
}

\begin{abstract}
RESUMEN:
El presente trabajo de investigación se realizó en la Clinica Veterinaria Basadre de la Escuela de Medicina Veterinaria y Zootecnia de la Universidad Nacional Jorge Basadre Grohmann de la ciudad de Tacna. El objetivo fue evaluar las anomalias congénitas y adquiridas de un total de 163 caninos, se diagnostico 123 anomalias congénitas y 40 anomalias adquiridas. Las anomalias congénitas según ubicación, displasia de cadera $8.1 \%(10)$, carcinoma de la glándula mamaria $6.5 \%$, (8), carcinoma prostático benigno $4.8 \%$ (6), carcinoma pulmonar $2.4 \%$ (3), carcinoma del higado $0.8 \%$ (1), anomalias adquiridas según ubicación, pio metra $25 \%(10)$, insuficiencia cardiaca $5 \%$ (2) fractura de fémur $12.5 \%$ (5), insuficiencia cardiaca $5 \%$ (2), fractura de tibia $2.5 \%$ (1), fractura de costilla $2.5 \%$, anomalias congénitas según sexo, displasia de cadera machos $2.4 \%$ (3) hembras $39.8 \%$ (7), carcinoma pulmonar machos $0.8 \%$ (1) hembras $1.6 \%(2)$, carcinoma de higado macho $0.85 \%$ (1), carcinoma mamario hembra $6.5 \%(8)$, carcinoma prostático machos 4.8(6), anomalias adquiridas, según sexo, pio metra hembra 10\%(10), fractura de fémur macho $10 \%(4)$ hembras $2.5 \%(1)$, fractura de tibia macho $2.5 \%(1)$, insuficiencia cardiaca macho $2.5 \%$ (1), fractura de costilla hembra $2.5 \%(1)$, anomalias congénitas según edad (años) displasia cadera 0-5, 7.3\% (9) 6-10, 0.8\% (1), carcinoma pulmonar $0-5,0.8 \%$ (1) 6-10, 1.6\% (2), carcinoma hígado 6-10, 0.85\% (1), carcinoma glándula mamaria 0-5, 0.8\% (1) $6-10,5.65 \%$ (7), carcinoma prostático $0-5,1.6 \%$ (2) 6-10, 3\% (2) osteosarcoma $0-5,0.8 \%$ (1) $6-10,2.4 \%$ (3) y anomalias adquiridas según edad, pio metra $0-5,5 \%(2) 6-10,20 \%$ (8), insuficiencia cardiaca $6-10,5 \%$ (2) fractura fémur $0-5,12.5 \%(5)$, fractura tibia $2.5 \%$ (1), fractura de costilla $6-10, g 2.5 \%(1)$.
\end{abstract}

Palabras Clave: Frecuencias patológicos, Anomalias Congénitas, Anomalias Adquiridas, Diagnostico imágenes, placas radiográficas.

\section{ABSTRACT:}

This research was carried out at the Veterinary Clinic Basadre School of Veterinary Medicine and Animal Science, in National University Jorge BasadreGrohmann city of Tacna. The objective was to assess congenital anomalies and acquired of a total of $163 \mathrm{dogs}, 123$ were diagnosed congenital anomalies and 40 of acquired abnormalities. Congenital abnormalities by location, hip dysplasia $8.1 \%$ (10), mammary gland carcinoma $6.5 \%$ (8), benign prostatic carcinoma $4.8 \%(6), 2.4 \%$ lung carcinoma (3), liver carcinoma $0.8 \%$ (1), acquired abnormalities by location, piometra $25 \%$ (10), heart failure, $5 \%$ (2) $12.5 \% \square \square$ femur fracture (5), heart failure, $5 \%$ (2) $2.5 \%$ tibial fracture (1) fracture $2.5 \%$ rib, congenital anomalies by sex, male hip dysplasia $2.4 \%$ (3) $39.8 \%$ females (7), lung carcinoma males $0.8 \%$ (1) $1.6 \%$ females (2), liver carcinoma male $0.85 \%$ (1), $6.5 \%$ female breast carcinoma (8), prostate carcinoma males $4.8(6)$, acquired anomalies, by sex, female metrapio $10 \%$ (10), male femur fracture $10 \%$ (4) females $2.5 \%$ (1), fracture male tibia $2.5 \%(1)$, heart failure male $2.5 \%(1)$, rib fracture female $2.5 \%(1)$, congenital anomalies by age (years) $0-5$ hip dysplasia, $7.3 \%$ (9) 6-10, 0.8\% (1), lung carcinoma $0-5,0.8 \%$ (1) $6.10,1.6 \%$ (2), liver carcinoma $6-10,0.85 \%$ (1), mammary carcinoma $0-5,0.8 \%$ (1) $6.10,5.65 \%$ (7), prostate carcinoma $0-5,1.6 \%$ (2) $6.10,3 \%$ (2) $0-5$ osteosarcoma, $0.8 \%$ (1) 10.6, 2.4\% (3) and acquired abnormalities by age, peep metra 0-5, 5\% (2) 6-10, 20\% (8), heart failure, 6-10, $5 \%$ (2) femur fracture $0-5,12.5 \% \square \square(5)$, tibia fracture $2.5 \%(1), 6-10$ rib fracture, $g 2.5 \%(1)$.

Keywords: Pathological Frequency, Congenital Abnormalities, Acquired Abnormalities, Diagnosticimaging, X-rays

Magister Fisiopatologia Médico Veterinario y Zootecnista Facultad Ciencias Agropecuarias. Universidad Nacional Jorge Basadre Grohmann. 


\section{INTRODUCCIÓN}

En la Clínica Veterinaria Basadre de la Universidad Nacional Jorge Basadre Grohmann de la ciudad de Tacna, se ha llevado a cabo el presente estudio de investigación, donde se ha evaluado las anomalías congénitas y adquiridas en caninos.

La frecuencia de presentación de Anomalías congénitas según edad fueron:

Displasia de cadera, Carcinoma pulmonar, Carcinoma de hígado, Carcinoma de la glándula prostática, y Osteosarcoma. Según sexo fueron; Displasia de cadera, Carcinoma pulmonar, Carcinoma de hígado, Carcinoma de la glándula prostática, y Osteosarcoma.

Para Anomalías adquiridas la frecuencia de presentación según edad son:

Piometra, Insuficiencia cardiaca, Fractura de fémur, Fractura de tibia y Fractura de costilla.

\section{MATERIALES Y MÉTODOS}

\subsection{Muestreo}

\subsubsection{Ubicación de la Zona de Muestreo:}

Clínica Veterinaria Basadre por cuadros clínicos, diferentes al motivo de estudio; tales como vacunaciones, cirugías, consultas, tratamientos, etc. Se tomaron imágenes radiológicas a aquellos animales sospechosos y fotografias para recopilar la información necesaria.

\subsubsection{Toma de Muestras y Traslado}

Para la evaluación de los animales según los casos se realizaron el diagnóstico de las imágenes de rayos $\mathrm{x}$ independientemente.

\subsubsection{Equipos y Materiales}

Rayos x

2.1.4.Método para evaluación de presentación de Anomalías congénitas y adquiridas:

Se realizó una observación directa y un examen externo de los animales, considerando la apariencia general y conformación utilizando a través la palpación, observación de las diferentes áreas topográficas del animal así se empleó la toma de placa y el respectivo revelado y fijación y la lectura de las anomalías, Displasia de cadera, Carcinoma pulmonar, Carcinoma de hígado, Carcinoma de la glándula prostática, y Osteosarcoma, a través del negatoscopio, los mismos que se anotaron en su historia clínica pre-elaborada.

\section{RESULTADOS:}

\subsection{Zona de estudio}

Tabla $\mathrm{N}^{0} 1$. Anomalías congénitas de caninos según edad mediante el diagnóstico de imágenes de rayos x

\begin{tabular}{|c|c|c|c|c|c|c|}
\hline \multirow{3}{*}{$\begin{array}{l}\text { ESPECIES } \\
\text { PERROS }\end{array}$} & \multirow[b]{2}{*}{ ANOMALAS } & \multirow{2}{*}{$\begin{array}{l}N^{\circ} \\
\text { animales }\end{array}$} & \multicolumn{2}{|c|}{ EDAD } & \multicolumn{2}{|l|}{$\%$} \\
\hline & & & $0-5$ & $6-10$ & $0-5$ & $6-10$ \\
\hline & DISPLASIA DE CADERA & 123 & 9 & 1 & 7.3 & 0.8 \\
\hline & CARCINOMA PULMONAR & & 1 & 2 & 0.8 & 1.6 \\
\hline & CARCINOMADE HIGADO & & & 1 & & 0.8 \\
\hline & $\begin{array}{l}\text { CARCINOMA DE LA } \\
\text { GLANDULAMAMARIA }\end{array}$ & & 1 & 7 & 0.8 & 5.6 \\
\hline & $\begin{array}{l}\text { CARCINOMA } \\
\text { PROSTATICO BENIGNO }\end{array}$ & & 2 & 4 & 1.6 & 3.2 \\
\hline & OSTEOSARCOMAS & & 1 & 3 & 0.8 & 24 \\
\hline \multicolumn{3}{|c|}{ PROMEDIOGENERAL } & 14 & 18 & & \\
\hline
\end{tabular}

En la Tabla $\mathbf{N}^{\circ} \mathbf{1}$. Se observa la presentación de Anomalías congénitas en canes, de una población total de 123 animales muestreados, 09 animales presentaron displasia de cadera entre las edades de $0-5$ años que representa el 7.3 y un caso entre las edades entre 6 a 10 años que representa el $0.8 \%$ seguido del carcinoma de la glándula mamaria con 7 casos entre las edades de 6 a 10 años que representa el $5.6 \%$, y un caso de 0 a 5 años que representa de $0.8 \%$, carcinoma prostático benigno, con 4 casos entre las edades de 6 a 10 años que representa el $3.2 \%$ y 2 casos entre las edades de 0 a 5 años y como valores intermedios se presentaron: el carcinoma pulmonar con 2 casos entre las edades de 6 a 10 años que representa el $1.6 \%$ y 1 caso entre las edades de 0 a 5 años que representa el $0.8 \%$, el carcinoma de hígado con 1 caso entre las edades entre 6 a 10 años, el osteosarcoma con 3 casos entre las edades de 6 a 10 años que representa el $2.4 \%$ y 1 caso entre las edades de 0 a 5 años que representa $0.8 \%$ respectivamente.

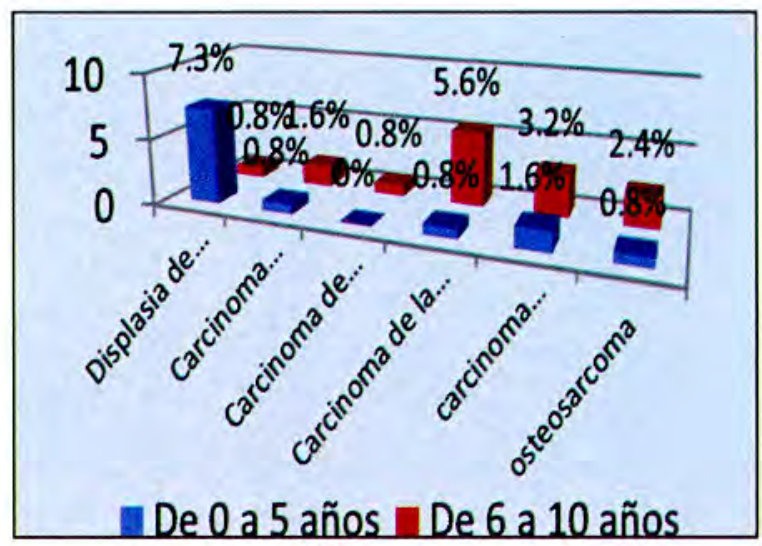

Figura $\mathrm{N}^{\circ} 1$. Anomalías congénitas de caninos según edad mediante el diagnóstico de imágenes de rayos $\mathrm{x}$

\section{Figura $\mathrm{N}^{\circ}$ 1. Anomalías congénitas en caninos.-}

Se observa con mayor representatividad la displasia de cadera con $7.3 \%$ seguido de carcinoma de glándula mamaria con $5.6 \%$. 
Tabla $N^{\circ}$ 2. Anomalías congénitas de caninos según sexo mediante el diagnóstico de imágenes de $\operatorname{rayos} x$

\begin{tabular}{|c|c|c|c|c|c|c|}
\hline \multirow{2}{*}{ ESPECIES } & \multirow{2}{*}{ ANOMALIAS } & \multirow{2}{*}{$\begin{array}{c}\mathrm{N}^{\circ} \\
\text { animales }\end{array}$} & \multicolumn{2}{|c|}{$\begin{array}{l}\mathrm{N}^{\circ} \mathrm{DE} \\
\text { CASOS }\end{array}$} & \multicolumn{2}{|c|}{$\%$} \\
\hline & & & M & $\mathrm{H}$ & $\mathbf{M}$ & H \\
\hline \multirow[t]{6}{*}{ PERROS } & $\begin{array}{l}\text { DISPLASIA DE } \\
\text { CADERA }\end{array}$ & 123 & 3 & 7 & 2.4 & 39.8 \\
\hline & $\begin{array}{l}\text { CARCINOMA } \\
\text { PULMONAR }\end{array}$ & & 1 & 2 & 0.8 & 1.6 \\
\hline & $\begin{array}{l}\text { CARCINOMA DE } \\
\text { HIGADO }\end{array}$ & & 1 & & 0.8 & \\
\hline & $\begin{array}{c}\text { CARCINOMA DE } \\
\text { LA GLANDULA } \\
\text { MAMARIA }\end{array}$ & & & 8 & & 6.5 \\
\hline & $\begin{array}{c}\text { CARCINOMA } \\
\text { PROSTATICO } \\
\text { BENIGNO }\end{array}$ & & 6 & & 4.8 & \\
\hline & OSTEOSARCOMAS & & 3 & 1 & 2.4 & $\begin{array}{lll}0.8 & \\
\end{array}$ \\
\hline \multicolumn{3}{|c|}{ PROMEDIO GENERAL } & 14 & 18 & & \\
\hline
\end{tabular}

En la Tabla $N^{\circ}$ 2. Se observa la presentación de Anomalías congénitas de caninos según sexo, de una población total de 123 animales evaluados, 7 hembras presentaron displasia de cadera que representa el 39.8\% y 3 casos en machos presentaron que representa el $2.4 \%$, seguido del carcinoma de la glándula mamaria con 8 casos en hembras que representa el $6.5 \%$ y el carcinoma prostático benigno con 6 casos que representa el $4.8 \%$ y con osteosarcoma 3 casos en machos que representa $2.4 \%$ y 1 caso en hembras que representa $0.8 \%$ y carcinoma pulmonar con dos casos en hembras que representa $1.6 \%$ y 1 caso en machos que representa el $0.8 \%$.

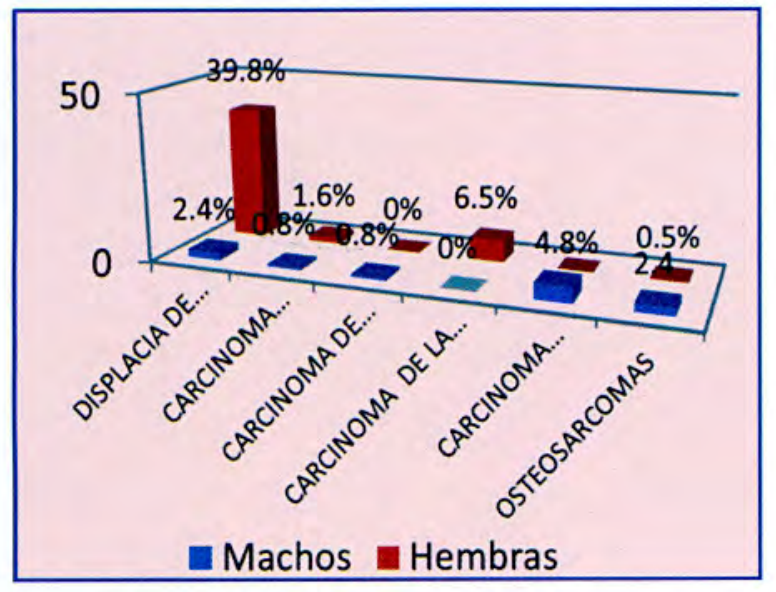

Figura $\mathrm{N}^{\circ} 2$. Anomalías congénitas de caninos según sexo mediante el diagnóstico de imágenes de rayos $\mathrm{x}$

Figura $\mathrm{N}^{\circ} 2$. Anomalías congénitas de caninos según sexo.- Se observa mayor representatividad la displasia de cadera en hembras con $39.8 \%$ y con menor representatividad el carcinoma pulmonar y carcinoma del hígado con $0.8 \%$ en machos.
Tabla $N^{\circ}$ 3. Anomalías congénitas de caninos según ubicación mediante el diagnóstico de imágenes de rayos $x$.

\begin{tabular}{|c|c|c|c|c|c|c|c|}
\hline \multirow[b]{2}{*}{ ESPECIES } & \multirow[b]{2}{*}{ ANOMALIAS } & \multirow{2}{*}{$\begin{array}{l}\mathrm{N}^{\circ} \\
\text { animales }\end{array}$} & \multirow{2}{*}{$\begin{array}{l}N^{\circ} \text { DE } \\
\text { CASOS }\end{array}$} & \multicolumn{4}{|c|}{ UBICACIÓN } \\
\hline & & & & A & $\mathrm{T}$ & $\begin{array}{l}\mathrm{A} \\
\%\end{array}$ & $\begin{array}{l}\mathrm{T} \\
\%\end{array}$ \\
\hline \multirow[t]{6}{*}{ PERROS } & $\begin{array}{l}\text { DISPLASIADE } \\
\text { CADERA }\end{array}$ & 123 & 10 & 10 & & 8.1 & \\
\hline & $\begin{array}{l}\text { CARCINOMA } \\
\text { PULMONAR }\end{array}$ & & 3 & & 3 & & 2.4 \\
\hline & $\begin{array}{l}\text { CARCINOMA DE } \\
\text { HIGADO }\end{array}$ & & 1 & 1 & & 0.8 & \\
\hline & $\begin{array}{l}\text { CARCINOMA DE } \\
\text { LAGLANDULA } \\
\text { MAMARIA } \\
\end{array}$ & & 8 & 8 & & 6.5 & \\
\hline & \begin{tabular}{|l|} 
CARCINOMA \\
PROSTATICO \\
BENIGNO \\
\end{tabular} & & 6 & 6 & & 4.8 & \\
\hline & OSTEOSARCOMAS & & 4 & 3 & 1 & 2.4 & 0.8 \\
\hline \multicolumn{3}{|c|}{ PROMEDIO GENERAL } & 32 & & & & \\
\hline
\end{tabular}

En la Tabla $\mathbf{N}^{\circ}$ 3. Se observa la presentación de Anomalias congénitas según ubicación en caninos, de una población total de 123 animales muestreados, 10 animales presentaron displasia de cadera a nivel abdominal que representa el $8.1 \%$, seguido del carcinoma de la glándula mamaria con 8 casos a nivel abdominal que representa $6.5 \%$ y carcinoma prostático benigno con 6 casos a nivel abdominal que representa $4.8 \%$, seguido del carcinoma pulmonar con 3 casos que representa el $2.4 \%$ y carcinoma del hígado con 1 caco a nivel abdominal que representa el $0.8 \%$.

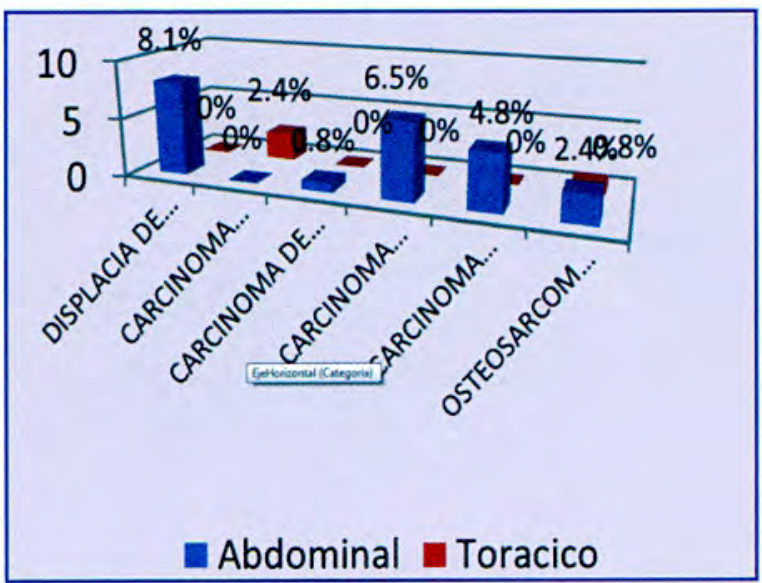

Figura $N^{\circ} 3$. Anomalías congénitas de caninos según ubicación mediante el diagnóstico de imágenes de rayos- $\mathrm{X}$

Figura $\mathrm{N}^{\circ}$ 3. Anomalías congénitas en caninos.- $\mathrm{Se}$ observa con mayor representatividad la displasia de cadera a nivel abdominal con $8.1 \%$ y con menor representatividad carcinoma de hígado con $0.8 \%$ a nivel abdominal. 


\section{MALFORMACIONES ADQUIRIDA}

Tabla $N^{\circ}$ 4. Anomalias adquiridas de caninos según edad mediante el diagnóstico de imágenes de rayos $\mathrm{x}$

\begin{tabular}{|c|c|c|c|c|c|c|}
\hline \multirow[b]{2}{*}{ ESPECIE } & \multirow[b]{2}{*}{ MALFORMACIONES } & \multirow[b]{2}{*}{$\begin{array}{l}\mathrm{N}^{\circ} \\
\text { animales }\end{array}$} & \multicolumn{4}{|c|}{ UBICACIÓN } \\
\hline & & & M & $\mathbf{H}$ & $\begin{array}{l}\mathrm{M} \\
\%\end{array}$ & $\begin{array}{l}\mathrm{H} \\
\%\end{array}$ \\
\hline PERROS & PIOMETRA & 40 & & 10 & & 10 \\
\hline & $\begin{array}{l}\text { INSUFICIENCIA } \\
\text { CARDIACA }\end{array}$ & & 1 & 1 & 2.5 & 2.5 \\
\hline & FRACTURA FEMUR & & 4 & 1 & 10 & 2.5 \\
\hline & FRACTURA TIBIA & & 1 & & & 2.5 \\
\hline & $\begin{array}{l}\text { FRACTURA } \\
\text { COSTILLA }\end{array}$ & & & 1 & & 2.5 \\
\hline
\end{tabular}

En la Tabla $\mathbf{N}^{\circ}$ 4. Se observa la presentación de anomalías adquiridas en caninos, de una población de 40 animales evaluados 8 presentaron pio metra entre las edades de 6 a 10 años que representa el $20 \%$, 2 casos entre las edades 0 a 5 años que representa el $5 \%$, seguido de fractura de fémur con 5 casos entre las edades de 0a 5 años el $12,5 \%$, insuficiencia cardiaca con 2 casos entre las edades de 6 a 10 años que representa el $5 \%$, y con menor frecuencia fractura de tibia entre las edades de 0 a 5 años que representa el $2.5 \%$ y fractura de costilla con 1 caso, entre las edades 6 a 10 años que representa $2.5 \%$.

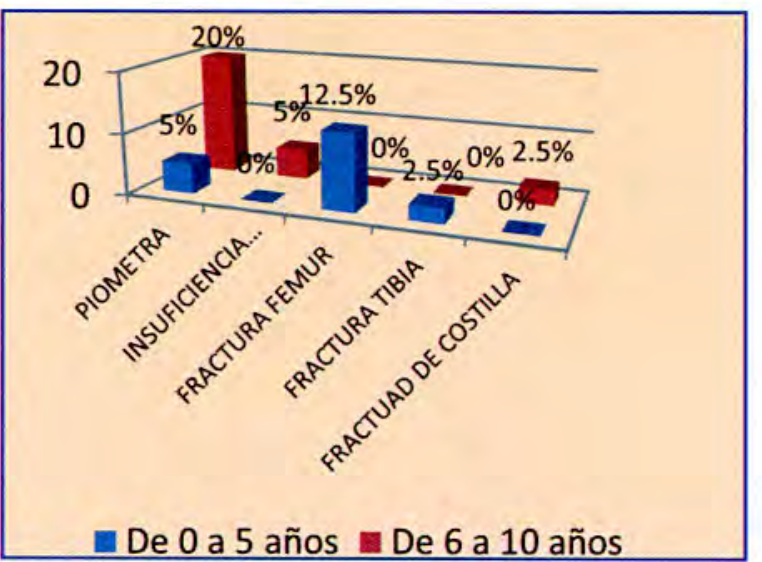

Figura $\mathbf{N}^{\circ} 4$. Anomalías adquiridas de caninos según edad mediante el diagnóstico de imágenes de rayos $\mathrm{x}$

Figura $\mathrm{N}^{\circ}$ 4. Anomalías Adquiridas en caninos.- $\mathrm{Se}$ observa con mayor representatividad la pio metra con $20 \%$ y con menor representatividad fractura de costilla con $2.5 \%$.

Tabla $\mathbf{N}^{\circ} \mathbf{5}$. Anomalías adquiridas de caninos según sexo mediante el diagnóstico de imágenes de rayos $\mathrm{x}$

\begin{tabular}{|l|l|l|l|l|l|l|}
\hline \multirow{2}{*}{ ESPECIES } & \multirow{2}{*}{ ANOMALIAS } & \multirow{2}{*}{$\begin{array}{l}N^{\circ} \\
\text { animales }\end{array}$} & \multicolumn{2}{|c|}{ EDAD } & \multicolumn{2}{|c|}{$\%$} \\
\cline { 4 - 8 } & & & $\mathbf{0 - 5}$ & $\mathbf{6 - 1 0}$ & $\mathbf{0 - 5}$ & $\mathbf{6 - 1 0}$ \\
\hline PERROS & PIOMETRA & 40 & 2 & 8 & $\mathbf{5}$ & $\mathbf{2 0}$ \\
\hline & $\begin{array}{l}\text { INSUFICIENCIA } \\
\text { CARDIACA }\end{array}$ & & & 2 & & $\mathbf{5}$ \\
\hline & $\begin{array}{l}\text { FRACTURA } \\
\text { FEMUR }\end{array}$ & & 5 & & $\mathbf{1 2 . 5}$ & \\
\hline & FRACTURA TIBIA & & 1 & & $\mathbf{2 . 5}$ & \\
\hline & $\begin{array}{l}\text { FRACTURA DE } \\
\text { COSTILLA }\end{array}$ & & 1 & & $\mathbf{2 . 5}$ \\
\hline
\end{tabular}

En la Tabla $\mathbf{N}^{\circ}$ 5. Se observa la presentación de Anomalías adquiridas de caninos según sexo, de una población de 40 animales evaluados, 10 hembras presentaron pio metra que representa el $10 \%$ y 4 machos presentaron fractura de fémur que representa el $10 \%$ y 1 hembra presento fractura de fémur que representa el $2.5 \%$ seguido de fractura de tibia un macho que representa el $2.5 \%$, seguido de insuficiencia cardiaca 1 caso en macho y hembra que representa el $2.5 \%$ y fractura de costilla 1 caso en de la hembra que representa el $2.5 \%$ respectivamente.

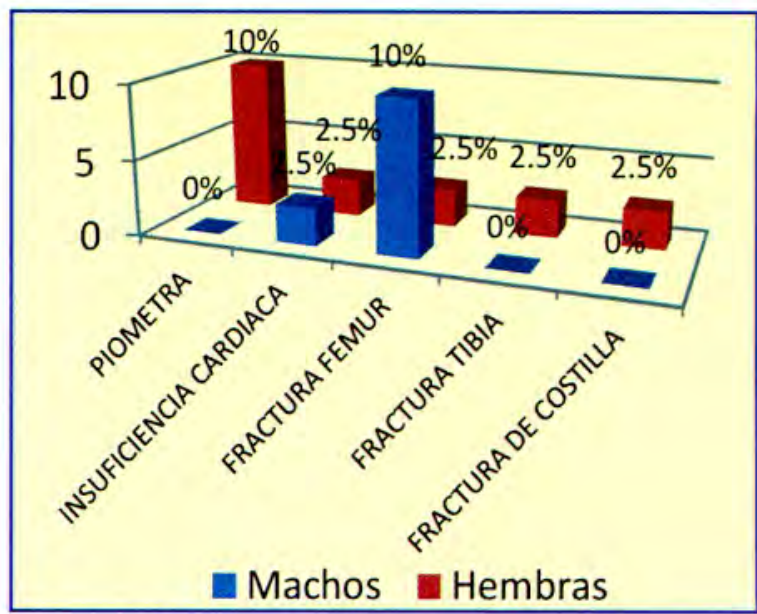

Figura $\mathbf{N}^{\circ} \mathbf{5}$. Anomalías adquiridas de caninos según sexo mediante el diagnóstico de imágenes de rayos $\mathrm{x}$

Figura $\mathbf{N}^{\circ}$ 5. Anomalías Adquiridas en caninos.- Se observa con mayor representatividad la pio metra en hembras con $10 \%$ y con menor representatividad fractura de costilla en hembras, fractura de tibia en machos e insuficiencia cardiaca en hembras con $2.5 \%$ respectivamente.

Tabla $\mathbf{N}^{\circ}$ 6. Anomalías adquiridas de caninos según ubicación mediante el diagnóstico de imágenes de rayos $\mathrm{x}$

\begin{tabular}{|c|c|c|c|c|c|c|}
\hline \multirow[b]{2}{*}{ ESPECIE } & \multirow[b]{2}{*}{ ANOMALIAS } & \multirow[b]{2}{*}{$\begin{array}{l}\mathrm{N}^{\circ} \\
\text { animales }\end{array}$} & \multicolumn{4}{|c|}{ UBICACION } \\
\hline & & & $\mathbf{A}$ & $\mathbf{T}$ & $\begin{array}{l}\mathbf{A} \\
\%\end{array}$ & $\begin{array}{l}\text { T } \\
\% \\
\end{array}$ \\
\hline \multirow[t]{5}{*}{ PERROS } & PIOMETRA & 40 & 10 & & 25 & \\
\hline & $\begin{array}{l}\text { INSUFICIENCIA } \\
\text { CARDIACA }\end{array}$ & & & 2 & & 5 \\
\hline & $\begin{array}{l}\text { FRACTURA } \\
\text { FEMUR }\end{array}$ & & 5 & & 12.5 & \\
\hline & FRACTURA TIBIA & & 1 & & 2.5 & \\
\hline & $\begin{array}{l}\text { FRACTURA DE } \\
\text { COSTILLA }\end{array}$ & & & 1 & & 2.5 \\
\hline
\end{tabular}

En la Tabla $\mathbf{N}^{\circ}$ 6. Se observa la presentación de Anomalías adquiridas en caninos según ubicación, de una población de 40 animales evaluados, 10 animales presentaron piometra a nivel abdominal que representa el $25 \%$, seguido de Fractura de fémur a nivel abdominal con 5 animales que representa el $12.5 \%$ y 2 animales presentaron insuficiencia cardiaca a nivel torácico que representa el $5 \%$ y fractura de tibia 1 caso a nivel abdominal que representa el $2.5 \%$ y 1 caso de fractura de costilla a nivel torácico que representa el $2.5 \%$ 


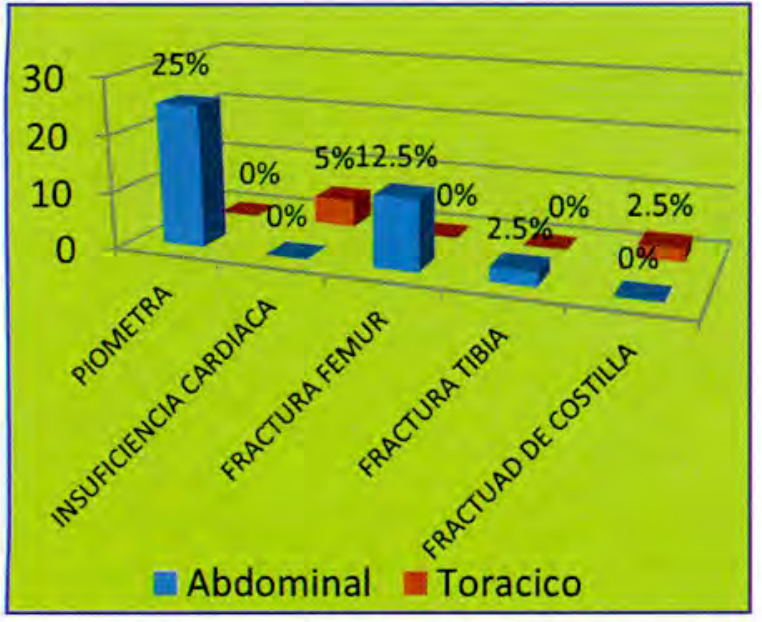

Figura $\mathbf{N}^{\circ}$ 6. Anomalías adquiridas de caninos según ubicación mediante el diagnóstico de imágenes de rayos $\mathrm{x}$

Figura $N^{\circ}$ 6. Anomalías adquiridas en caninos.- Se observa con mayor representatividad la piometra con $25 \%$ a nivel abdominal y con menor representatividad, fractura de tibia con $2.5 \%$ a nivel abdominal respectivamente.

\section{IV.DISCUSIÓN}

\section{Anomalías congénitas}

Los resultados obtenidos de los diferentes casos diagnosticados en la Clínica Veterinaria Basadre, con mayor frecuencia es la displasia de cadera según sexo en hembras presento el $39.8 \%$, y según la ubicación a nivel abdominal con $8.1 \%$, seguido de la edad con $7.50 \%$ entre la edades de $0-5$ años. Sin embargo Dávila F.R en el año 2002, encontró valores mayores al presente estudio, $55 \%$ de displasias en caninos de 1 a 1.5 años de edad, en la raza Rottweiler, es decir el sexo y la edad no fueron factores de riesgo para la presentación de displasia de cadera.

Según Donald L.1999. Indica los porcentajes de incidencia varía entre $0.9 \%$ en perros de raza Borzoi, $47 \%$ en San Bernardo, evaluados radiográficamente; los resultado del presente estudio se encuentran dentro el rango. El carcinoma de la glándula mamaria presentó $5.6 \%$ entre las edades de 6 a 10 años, según su ubicación se encontró $6.5 \%$ a nivel torácico, resultados menores encontró Moulton, 1990 con $25 \%$ a un $50 \%$ de todas las neoplasias caninas; Brodey, R.S. et al. En el año 1983 Encontró igualmente, $50 \%$ de los tumores son malignos.

Carcinoma Prostático. Los resultados obtenidos fueron $3.2 \%$ entre las edades de 6 a 10 años, y $4.8 \%$ según la ubicación a nivel abdominal: en casos de hiperplasia, $22 \%$ en prostatitis y sólo $12 \%$ en glándulas neoplásicas, resultados similares encontró Asurdia, P 1998 en Guatemala.

\section{Anomalías adquiridas}

De los resultados obtenidos en el presente estudio, 40 animales evaluados 8 presentaron pio metra entre las edades de 6 a 10 años que representa el $20 \%$; resultados similares encontró Saunders Co. En el año 1985 que indica la pio metra, se puede presentar en perras de cualquier edad después de su primer celo, aunque tienen mayor predisposición las perras mayores de 5 años 8 semanas, después del último estro. El rango de edad en que se pueden ver afectadas las perras varía de 6 meses a 16 años; sin embargo Bowen y coks, en el año 1985. Ha demostrado que el $25 \%$ de las perras que reciben estrógenos para evitar la implantación post monta desarrollan pió metra.

La Fractura de fémur en 5 animales se presentó entre las edades de 0 a 5 años que representa el $12,5 \%$ y 4 machos presentó el $10 \%$, resultados similares encontró Rodríguez D en el año 2007. En diagnóstico radiográfico de un total de 25 casos en perros (politraumatismo) fue la fractura femoral, de los cuales 9 eran menores de un año que hacen el $36 \%$, y $14 \%$ entre las edades de 2 a 7 años.

Igualmente Donald. L. en el año 1999 encontró la incidencia de las fracturas de fémur en un $45 \%$ de todas las fracturas de huesos largos.

Referente a la insuficiencia cardiaca 2 casos se encontró en el presente estudio entre las edades de 6 a 10 años que representa el 5\%; resultados similares encontró Trigo en el año 1995, además indica que la insuficiencia cardiaca es una alteración pato fisiológica en la que la función cardiaca anormal sistólica o diastólica es responsable de la incapacidad del corazón para mantener presiones de llenado ventricular normales o de impulsar la sangre a la frecuencia suficiente para el mantenimiento del metabolismo tisular.

\section{V.-CONCLUSIONES}

Según los resultados obtenidos en el presente trabajo de investigación, podemos concluir que:

1.- Según edad, 09 casos presentaron displasia de cadera entre las edades de 0-5 años un caso entre las edades de 6-10 años.

2.- Según sexo, 7 casos en hembras presentaron displasia de cadera.

3.- Según ubicación, 10 casos presentaron displasia de cadera a nivel abdominal.

4.- Según edad, 8 casos presentaron pio metra entre las edades de 6 a 10 años.

5.- Según sexo, 10 casos en hembras presentaron pio metra.

6.- Según ubicación, 10 casos presentaron pío metra a nivel abdominal. 


\section{REFERENCIAS BIBLIOGRÁFICAS.}

1. Álvarez, F. 2002. Neoplasias cutáneas. (en línea). Consultado 29 feb. (2004). Disponible en .com/ cyber/neoplasias_cutaneas.ph

2 Azurdia, P. (1998). Estudio retrospectivo y prospectivo de tumores en perros. Tesis Med. Vet. Guatemala, Universidad de San Carlos de Guatemala, Facultad de Medicina Veterinaria y Zootecnia. $99 \mathrm{p}$.

3. Birchard, S; Sherding, R. (1996). Manual clinico de pequeñas especies. Trad. S. Lara México, D. F Interamericana. $1747 \mathrm{p}$.

4. Bonagura, J. (1997). Kirk; Terapéutica veterinaria de pequeños animales. Trad. J. Orizaga. 12ed. México, D. F., Interamericana. $1638 \mathrm{p}$.

5. Correa, J. (2000). Teratoma vs mielomeningocele. (en linea). Consultado 8 mayo 2004. Disponible en http://www.dynabizvenezuela.com/ images/dynabiz/103749/siteinfo/02\%20posteres\%mart es.pdf

6. Dávila F.R (2002) tesis de la UNMSM Frecuencia de displasia de cadera en caninos Rottweiler mayores de un añodeedad.

7. Donal L. (1999) Manual de ortopedia y reparación de fracturas de pequeños animales III Edición. Editorial Interamericana, Madrid España.

8. El Manual Merck de veterinaria. (1997). 2ed. Estados Unidos, Merck. 1386 p.
9. Ettinger, S. (1989). Textbook of veterinary internal medicine; diseases of the dog and cat. 3ed. United States, Saunders. 1199 p.

10. García, C. (2001). Curso de oncología en pequeñas especies. (en línea). Consultado el 15 mayo 2004. Disponible en http://ammvepe.com/cursodeon cologia.pdf

11. Goldman, A. (2003) (a). Abc de oncología. (en linea). Consultado 22 feb. 2004. Disponible en http:// www.mascotia.com/articulos/1196.ht

12. Holzworth, J. (1987). Diseases of the cat; medicine \& surgery. United States, Saunders. $971 \mathrm{p}$.

13. Iturriaga, M. (2003). Convulsiones en gatos. (en linea). Consultado 15 abr. 2004. Disponible en http://www. mevepa.cl/modules.php?=name=News $\&$ file $=$ article \&sid $=145$

14. LeMarie, J. (1995). Mastocitoma. (en línea). Consultado 29 feb. 2004. Disponible en http://www.seleccionesveterinarias.com/articulos/art4 $1 \mathrm{htm}$

15. Lujan, E. (2002). Tumores cutáneos II. (en linea). Consultado 22 feb. 2004. Disponible en articulos/876.htm

\section{Correspondencia:}

Hugo Flores Aybar

Ciudad Universitaria Fundo "Los Granados"

Av. Miraflores s/n. Tacna. Perú.

mvz-hugo@hotmail.com 